\title{
Myocardial steatosis, impaired energetics and reduced circumferential strain are early manifestations of diabetic cardiomyopathy and precede structural changes
}

\author{
Eylem Levelt ${ }^{1,2^{*}}$, Masliza Mahmod ${ }^{1}$, Camilla Wainwright ${ }^{1,2}$, Stefan K Piechnik ${ }^{1}$, Jane M Francis ${ }^{1}$, Jurgen E Schneider ${ }^{1}$ \\ , Paul Leeson ${ }^{3}$, Theodoros D Karamitsos ${ }^{1}$, Cameron Holloway ${ }^{1,2}$, Kieran Clarke², Stefan Neubauer ${ }^{1}$
}

From 17th Annual SCMR Scientific Sessions

New Orleans, LA, USA. 16-19 January 2014

\section{Background}

Patients with diabetes mellitus (DM) of longer duration have an increased incidence of heart failure, independent of hypertension and coronary disease, as shown in large population based studies. Mechanisms of diabetic cardiomyopathy are multifactorial and not fully elucidated. Cardiovascular magnetic resonance (CMR) and magnetic resonance spectrocopy (MRS) provide a noninvasive assessment of the functional, structural and metabolic status of the heart. Here we assessed whether subclinical functional, structural and metabolic alterations can be detected using MRI in a patient cohort of early-onset, stable and uncomplicated type $2 \mathrm{DM}$.

\section{Objective}

The aim of this study was to assess the earliest manifestations of diabetic cardiomyopathy using multiparametric CMR and MRS in a cohort of uncomplicated type $2 \mathrm{DM}$ patients with a short duration of disease.

\section{Methods}

13 patients (6 female, mean age $55 \pm 9$ years) with earlyonset (median 3 [IQR: 1-4] years) type $2 \mathrm{DM}$ and 10 healthy volunteers ( 5 female, mean age $57 \pm 9$ years) were enrolled. Patients were either drug naive for diabetic therapy or on treatment with metformin monotherapy, HBA1c $\geq 6.7$ and $\leq 8 \%$, with no history of coronary artery disease or uncontrolled hypertension.

'University of Oxford Centre for Clinical Magnetic Resonance Research, University of Oxford, Oxford, UK

Full list of author information is available at the end of the article
Myocardial lipid content and $\mathrm{PCr} / \mathrm{ATP}$ ratios were quantified using $1 \mathrm{H}$ - and 31P MRS, respectively. CMR included cine, tagging and native T1 mapping was performed at 3.0 T. LV diastology was characterised using echocardiography.

\section{Results}

Diabetic patients were well-matched with controls (Figure 1). Myocardial energetics were disturbed ( $\mathrm{PCr} /$ ATP ratio: $1.44 \pm 0.35$ vs. $1.98 \pm 0.18, \mathrm{p}<0.001)$ and myocardial triglyceride content increased $(1.06 \pm 0.61$ vs. $0.48 \pm 0.24 \%, \mathrm{p}=0.01$ ), despite the relatively short disease duration. Peak systolic circumferential strain was reduced, indicating subtle regional LV dysfunction. However, left ventricular volumes, mass and ejection fraction, as well as echocardiographic indices of diastolic function were similar in both groups. Furthermore, despite the metabolic abnormalities observed in diabetics, there was no difference in native $\mathrm{T} 1$ values (as a measure of myocardial fibrosis) between diabetic patients and controls $(1181 \pm 28 \mathrm{~ms}$ vs. $1195 \pm 33 \mathrm{~ms}$, $\mathrm{p}=0.30)$.

\section{Conclusions}

Abnormal myocardial energy metabolism, cardiac steatosis and reduced LV strain are present in uncomplicated type $2 \mathrm{DM}$ patients with a short duration of disease and precede the development of structural or other functional changes. CMR is a sensitive, non-invasive tool for assessment of myocardial pathophysiology, and may be helpful in the comprehensive phenotyping and staging of myocardial involvement in DM. 


\begin{tabular}{|c|c|c|c|}
\hline & $\begin{array}{c}\text { Type } 2 \text { Diabetes } \\
\text { Mellitus } \\
(n=13)\end{array}$ & $\begin{array}{l}\text { Controls } \\
(n=10)\end{array}$ & $P$ value \\
\hline \multicolumn{4}{|c|}{ Demographic features and clinical status } \\
\hline Age, years & $55 \pm 9$ & $57 \pm 9$ & 0.53 \\
\hline Male, n (\%) & $7(54)$ & $5(50)$ & \\
\hline Body Mass Index $\left(\mathrm{kg} / \mathrm{m}^{2}\right)$ & $30 \pm 5$ & $28 \pm 4$ & 0.24 \\
\hline Systolic BP (mmHg) & $130 \pm 9$ & $128 \pm 9$ & 0.61 \\
\hline Diastolic BP (mmHg) & $78 \pm 8$ & $74 \pm 6$ & 0.24 \\
\hline Heart rate, bpm & $70 \pm 15$ & $69 \pm 9$ & 0.76 \\
\hline \multicolumn{4}{|c|}{ Echocardiographic features } \\
\hline Mitral in-flow E/A ratio & $0.91 \pm 0.26$ & $0.91 \pm 0.18$ & 0.96 \\
\hline Tissue Doppler septal E/E' ratio & $9.6 \pm 3.1$ & $9.4 \pm 2.4$ & 0.88 \\
\hline \multicolumn{4}{|c|}{ Laboratory results } \\
\hline HBA1c, \% & $7.29 \pm 0.49$ & - & \\
\hline Triglyceride, $\mathrm{mmol} / \mathrm{L}$ & $1.74 \pm 0.74$ & $0.85 \pm 0.21$ & 0.06 \\
\hline Non-esterified fatty acids, $\mathrm{mmol} / \mathrm{L}$ & $0.65 \pm 0.47$ & $0.41 \pm 0.33$ & 0.28 \\
\hline \multicolumn{4}{|c|}{ CMR and MRS findings } \\
\hline LVEDV, $\mathrm{ml}$ & $125 \pm 21$ & $145 \pm 39$ & 0.12 \\
\hline LVESV, $\mathrm{ml}$ & $37 \pm 9$ & $47 \pm 17$ & 0.08 \\
\hline LVEF, \% & $70 \pm 6$ & $68 \pm 5$ & 0.35 \\
\hline LV Mass indexed to $\mathrm{BSA}, \mathrm{g} / \mathrm{m}^{2}$ & $67 \pm 15$ & $66 \pm 13$ & 0.35 \\
\hline LV Wall thickness, mm & $11 \pm 2$ & $9 \pm 1$ & 0.14 \\
\hline $\begin{array}{l}\text { Mid short axis peak systolic } \\
\text { circumferential strain }\end{array}$ & $-16 \pm 3$ & $-19 \pm 3$ & 0.05 \\
\hline $\begin{array}{l}\text { Peak circumferential diastolic strain rate, } \\
\mathrm{s}^{-1}\end{array}$ & $62 \pm 25$ & $74 \pm 13$ & 0.180 \\
\hline PCr/ATP ratio & $1.44 \pm 0.35$ & $1.98 \pm 0.18$ & $<0.001$ \\
\hline Myocardial lipid content, \% & $1.06 \pm 0.61 *$ & $0.48 \pm 0.24 *$ & $0.01^{*}$ \\
\hline Native myocardial T1 value, ms & $1181 \pm 28$ & $1195 \pm 33$ & 0.30 \\
\hline
\end{tabular}

Continuous data are mean \pm standard deviation, unless otherwise stated.

ATP, adenosine triphosphate; HBA1c, glycated haemoglobin A1c; LV, left ventricle/ventricular; LVEDD, left ventricular end-diastolic dimension; LVEF, left ventricular ejection fraction; LVESD, left ventricular end-systolic dimension; $\mathrm{PCr}$, phosphocreatine

Figure 1

\section{Funding}

'The National Institute for Health Research Oxford Biomedical Research Council and Hoffmann-La Roche supported this work.
doi:10.1186/1532-429X-16-S1-0114

Cite this article as: Levelt et al:: Myocardial steatosis, impaired

energetics and reduced circumferential strain are early manifestations

of diabetic cardiomyopathy and precede structural changes. Journal of

Cardiovascular Magnetic Resonance 2014 16(Suppl 1):O114.

\section{Authors' details}

${ }^{1}$ University of Oxford Centre for Clinical Magnetic Resonance Research,

University of Oxford, Oxford, UK. ²Department of Physiology, Anatomy and Genetics, University of Oxford, Oxford, UK. ${ }^{3}$ Oxford Cardiovascular Clinical

Research Facility, University of Oxford, Oxford, UK.

Published: 16 January 2014 